\title{
Rapid detection of quantum dot immune chromatography nasopharyngeal carcinoma EBNA1 antibody
}

\author{
JIAN HE, JING XU, PING WU, LINGFENG LIAO, HUATAO QUAN, \\ JING KANG, YONGQUAN TIAN and YAOYUN TANG \\ Department of Otorhinolaryngology Head and Neck Surgery, \\ Xiangya Hospital of Central South University, Changsha, Hunan 410008, P.R. China
}

Received August 3, 2017; Accepted December 6, 2017

DOI: $10.3892 / \mathrm{ol} .2018 .7729$

\begin{abstract}
This study explored the $605 \mathrm{~nm}$ carboxyl of water-soluble quantum dots to assess the practicability of the nasopharyngeal carcinoma marker EBNA1 antibody. We used $605 \mathrm{~nm}$ carboxyl water-soluble quantum dots and nasopharyngeal carcinoma EBNA1 antigen in 1-(3-dimethylaminopropyl-3-2-ethylcarbonimine hydrochloride to generate quantum dot-labeled antigen. Gel imaging system showed that serum group and 60 patients with nasopharyngeal carcinoma had significant differences in 30 cases of normal adult serogroup brightness, Levene test in the two groups ELISA absorbance value was $\mathrm{P}<0.001$, namely the nasopharyngeal carcinoma group and the normal adult serum group measured absorbance value difference was obvious. The statistical significance, and the detection technology were of high specificity and sensitivity. In conclusion, this study adopted double antigen clip combining immune chromatography test EBNA1 antibody in serum, compared with the traditional enzyme-linked immunoassays this method is more rapid, with simpler operation, rapid detection for developing quantum dot immune chromatography technology the EBNA1 antibody kit provides a theoretical basis.
\end{abstract}

\section{Introduction}

Quantum dots (QD), also known as semiconductor nanocrystals, mainly consist of elements including II-IV group elements (Cdse and Cds) or III-V group elements (InP and InAs). The particle is smaller than $100 \mathrm{~nm}$ in diameter. Due to the presence of crystal core, QD can emit specific fluorescence (1,2). In order to combine with other biological protein molecules when applying in medical field, amino groups or carboxyl groups can often be seen on the surface of QD (3). QD modification

Correspondence to: Dr Yaoyun Tang, Department of Otorhinolaryngology Head and Neck Surgery, Xiangya Hospital of Central South University, 87 Xiangya Road, Changsha, Hunan 410008, P.R. China

E-mail: y335r7@163.com

Key words: quantum dots, nasopharyngeal carcinoma, immune chromatography, EBNA1 mainly uses static adsorption, covalent coupling, thiol exchange and other methods to modify antibodies, peptides and oligonucleotides onto the surface of QD, where covalent coupling is the most common and stable method. Compared to the traditional organic molecular fluorescent materials, QD has the following advantages: i) wide and continuous excitation spectrum, with large absorption coefficient and high quantum yield; ii) narrow and symmetric emission spectrum without trailing; iii) strong fluorescence intensity and high signal to noise ratio; iv) good light stability and strong ability to resist photobleaching; v) emission wavelength changes with particle size. Owing to its unique chemical and optical properties, QD has gradually been applied in tumor biological medicine, such as prostate cancer (4), pancreatic cancer (5), breast cancer (5), liver cancer (6), colorectal cancer (7) and nasopharyngeal carcinoma (NPC) (8). This study aimed to explore the $605 \mathrm{~nm}$ carboxyl of water-soluble quantum dots to assess the practicability of the NPC marker EBNA1 antibody.

NPC is a common malignant tumor in the Southern Coast, Western Hunan and other places of China (9). Its incidence is up to $30 / 100,000$. It is the most frequent malignant tumor of otolaryngology head and neck surgery. Due to the complex nasopharyngeal anatomy, NPC is difficult to detect in early stage and only $10-20 \%$ of the patients are diagnosed at the early stage. Once there appear obvious symptoms, the patients are mainly at middle-to-advanced stage, so it is crucial to early diagnose NPC (10). The main causes of diagnostic difficulty in NPC are the atypical symptoms and no proper biopsy. There is a certain correlation between NPC and EB virus infection (11), so it is a main measurement for early NPC diagnosis in clinic to detect serum EB virus infection (such as EB-VCA IgA, EBNA1, Zta-IgA, EA/IgA and EBV-DNA) of NPC patients (12-14). EBNA1 is expressed in all of the NPC patients. It is the only virus core protein in NPC, so its change may be one of the cancer-promotion factors (15). Studies have found that EBNA1 may block NPC cell cycle at stage G1 through in vitro induction, thus blocking the growth of NPC cells. Currently, enzyme-linked immunosorbent assay (ELISA) qualitative analysis is the major method to detect EBNA1 antibody in clinic (16), but it still has the following disadvantages: i) ELISA kits are produced by different manufacturers and there is no unified standard; ii) ELISA method is complicated in use, so the results are affected by the operators, which is 
not likely to be carried out in grass-roots hospitals; iii) ELISA method is unable to realize rapid detection and moreover it is qualitative analysis.

Therefore, water-soluble carboxylated QD was used as the luminescent material in this study. Coupled with EBNA1 by covalent cross-linking method, the feasibility of $605 \mathrm{~nm}$ water-soluble carboxylated QD to label EBNA1 was explored and reported.

\section{Materials and methods}

Materials and reagents. Water-soluble (605 $\mathrm{nm})$ carboxylated QD was purchased from Jiayuan Quantum Dots Co., Ltd. (Wuhan, Hubei, China). Crosslinking agent, 1-(3-dimethylaminopropyl)-3-ethylcarbodiimide hydrochloride (EDC) was purchased from Sigma (Jiayuan, China). EBV EBNA1 antigen and positive standard of EBV EBNA1 antibody were purchased from Abcam (Cambridge, UK). Other common reagents were purchased from the Otolaryngology Laboratory of Xiangya Hospital of Central South University (Hunan, China).

All healthy control blood samples were obtained from volunteers, who were the permanent residents in Hunan province, China. All NPC samples were taken from NPC patients hospitalized in Xiangya Hospital of Central South University from May, 2012 to December, 2012. The patients were recorded for personal basic information and clinical data (age, sex, hospital, department, admission number, pathological diagnosis, clinical stage, main findings, ID number, native place, home address and phone number), as shown in the spreadsheet. All the samples were verified by the patients' consent and informed consent was signed. The study was approved by the Ethics Committee of Xiangya Hospital of Central South University.

Serum collection. i) A $5 \mathrm{ml}$ serum collection tube was used to collect fasting venous blood. The tube was filled with the blood as full as possible, for $\sim 3-4 \mathrm{ml}$, and then slightly turned upside down 3 times; ii) the tube was placed at room temperature for $1 \mathrm{~h}$; iii) the tube was centrifuged $(1,600 \mathrm{x} \mathrm{g})$ for $10 \mathrm{~min}$ at $4^{\circ} \mathrm{C}$; iv) the upper layer of the serum was carefully removed to $\mathrm{i}$ ). The $5 \mathrm{ml}$ cryogenic tube was placed on ice, $\sim 600 \mu \mathrm{l} \times 2$ tubes. The tubes were numbered and labeled, and preserved at $-80^{\circ} \mathrm{C}$.

Instruments and equipments. UV-VIS spectrophotometer (UV-2550; Shimadzu, Kyoto, Japan); LS-55 fluorescence spectrophotometer (Perkin Elmer, Waltham, MA, USA); ultrafiltration tube (Millipore, Billerica, MA, USA); desalting column (GE Healthcare, Piscataway, NJ, USA); immune chromatography test paper (Wuhan Jiayuan Quantum Dots Co., Ltd., Wuhan, China); gel imaging system (Alpha Imager HP).

Sample pretreatment. EBV EBNA1 antigen (Abcam) at $-20^{\circ} \mathrm{C}$, was thawed at room temperature. It was dissolved in the buffer solution (20 mM borate, $\mathrm{pH}$ 7.4). Ultrafiltration tube with molecular cut off of $10 \mathrm{kDa}$ was used to remove impurities, and final sample concentration was $2.2 \mathrm{mg} / \mathrm{ml}$ (Table I).

Labeling method. Covalent cross-linking was carried out on water-soluble carboxylated QD (Q3605) and EBV EBNA1 antigen under EDC to form QD-labeled antigen. Ultrafiltration
Table I. Sample amount.

\begin{tabular}{lccc}
\hline Antigen & $\begin{array}{c}\text { Antigen/ } \\
\text { QD }\end{array}$ & $\begin{array}{c}\text { Maximum emission } \\
\text { peak }\end{array}$ & $\begin{array}{c}\text { Full width at } \\
\text { half maximum }\end{array}$ \\
\hline Solution & $10: 1$ & $605 \mathrm{~nm}$ & $24 \mathrm{~nm}$ \\
Solution & $40: 1$ & $605 \mathrm{~nm}$ & $24 \mathrm{~nm}$ \\
\hline
\end{tabular}

QD, quantum dots.

tube with molecular cut off of $100 \mathrm{kDa}$ was used to remove unreacted antigen and other impurities, and final product was stored in $50 \mathrm{mM}$ borate at $\mathrm{pH} 8.4$ with $0.05 \% \mathrm{NaN}_{3}$.

Experimental procedure. i) Assemble of immune chromatography test paper: chromatography pad, nitrocellulose membrane (NC membrane) and absorbent filter paper were pasted on the viscous base in turn. Biodot strip cutting machine was used to cut the blank test paper into strips of $3 \mathrm{~mm}$ width. ii) Envelope of antigen: $0.5 \mu \mathrm{l}$ EB NA1 antigen was removed from NC membrane. Until the humidity was $<20 \%$, the membrane was placed in an oven at $25^{\circ} \mathrm{C}$ for $2 \mathrm{~h}$. iii) Detection buffer preparation: Antibody dilution was used to dilute the QD-labeled antigen as the detection buffer, and $75 \mu \mathrm{l}$ was removed to the microplate. iv) Sample loading: $5 \mu \mathrm{l}$ serum from each of the 60 NPC patients and 30 healthy people and positive samples of different concentrations, were mixed with detection buffer. Test paper in (ii) was inserted into microplate of (iii). The liquid will go upwards along the strip due to capillary action (5). It was observed under UV after $6 \mathrm{~min}$. If there was a positive reaction, orange fluorescence was observed under UV. The higher the EBNA1 antibody content was, the stronger the fluorescence was. However, if there was no corresponding antibody, then there was no fluorescence or just a weaker one. The findings were observed and imaged by gel imaging system with UV light. ELISA absorbency value was measured at the same time. SPSS 13.0 (SPSS Inc., Chicago, IL, USA) was used to obtain ROC of absorbance to calculate the specificity and sensitivity.

\section{Results}

QD fluorescence emission spectrum showed that fluorescence emission peak reached the peak around $605 \mathrm{~nm}$ with the peak height of 380 (Fig. 1). Agarose gel electrophoresis showed that the stable covalent bond was formed between water-soluble carboxyl QD and EBV EBNA1 antigen by amino and carboxyl condensation on the surface. Water-soluble carboxyl QD and EBV EBNA1 antigen were successfully linked suggesting a successful labeling.

There were significant differences between NPC patients and normal controls in QD-labeled fluorescence intensity (Table II).

Area under the curve (AUC) was 0.929 (95\% CI, 0.871-0.987), which had statistically significant difference compared to $\mathrm{A}=0.5(\mathrm{P}<0.001)$. The most upper and left point on curve in Fig. 2 was selected and Youden index was calculated according to coordinates of each point in SPSS analysis. With the maximum tangent point of Youden index as the critical point, the optimal threshold of FLISA fluorescence value in 
Table II. ELISA absorption results of NPC patients and normal people.

\begin{tabular}{lcccc}
\hline Group & Minimum & Maximum & Mean (X) & Standard deviation (S) \\
\hline Serum absorption of NPC patients & 0.032044 & 3.217905 & 0.97469163 & 0.667895186 \\
Serum absorption of normal people & 0.085533 & 0.690848 & 0.17310760 & 0.159114266 \\
\hline
\end{tabular}

NPC, nasopharyngeal carcinoma.
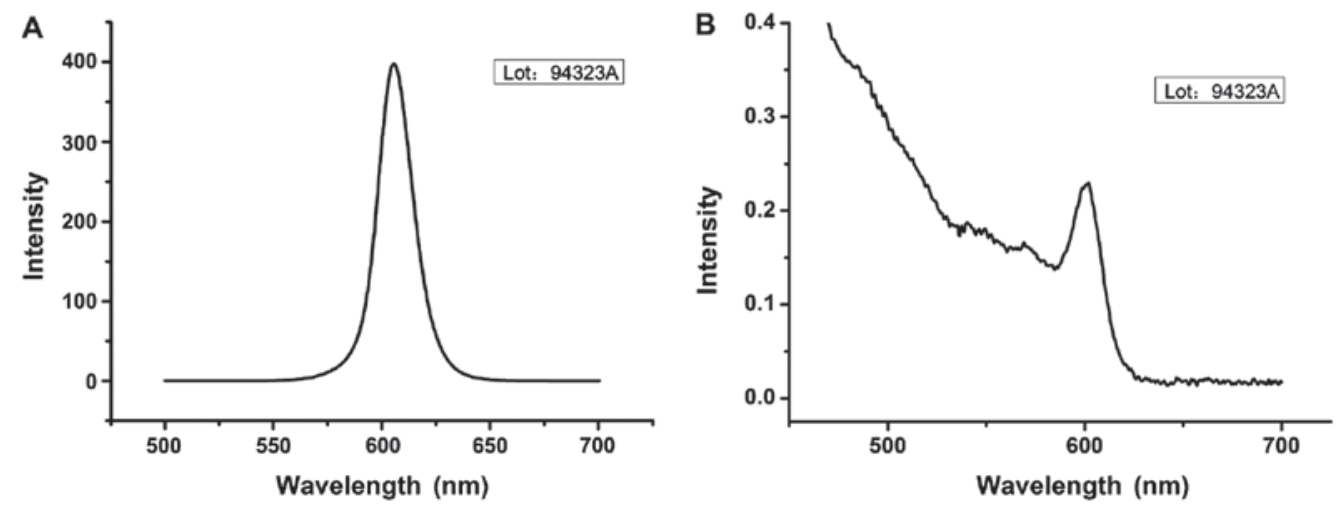

Figure 1. Fluorescence emission (A) and UV-VIS absorption (B) spectra of quantum dot (QD)-labeled EBNA1 antigen.

Table III. Sensitivity and specificity of minimum and maximum values of doubtful range.

\begin{tabular}{lcc}
\hline Cut-off point & Sensitivity & Specificity \\
\hline 1.000988 & 0.867 & 0.933 \\
1.091343 & 0.933 & 0.767 \\
\hline
\end{tabular}

the diagnosis of NPC was 0.680817 , with sensitivity of 0.917 , specificity of 0.900 and Youden index of 0.817 .

A smooth curve was drawn by hand above ROC (fold line) obtained from SPSS, which was tried to overlap the original line as far as possible. Then it was easy to see the general trend of ROC, which was steep in the bottom, slightly flat in the middle and flat in the upper part. In the lower part of ROC, the true positive rate increased quickly while the false positive rate more slowly. After turning point $(0.867$ and 0.867$)$, the true positive rate slowed down from the vertical jump, while the false positive rate accelerated, suggesting the two groups of people had large overlap; Again, after turning point (0.933 and 0.233 ), true positive rate slowed down again, while false positive rate fastened, suggesting the overlapping of the two groups of people was decreased. Therefore, dubious value should be selected between the two turning points, where there was considerable overlapping. According to statistical results, the corresponding cut-off points were 1.000988 and 1.091343, the doubtful range was 1.000988 and 1.091343). Sensitivity and specificity of the two points are shown in Table III.

\section{Discussion}

Based on an association between NPC and EB virus infection, detection of serum EB virus antibodies (e.g., EB-VCA

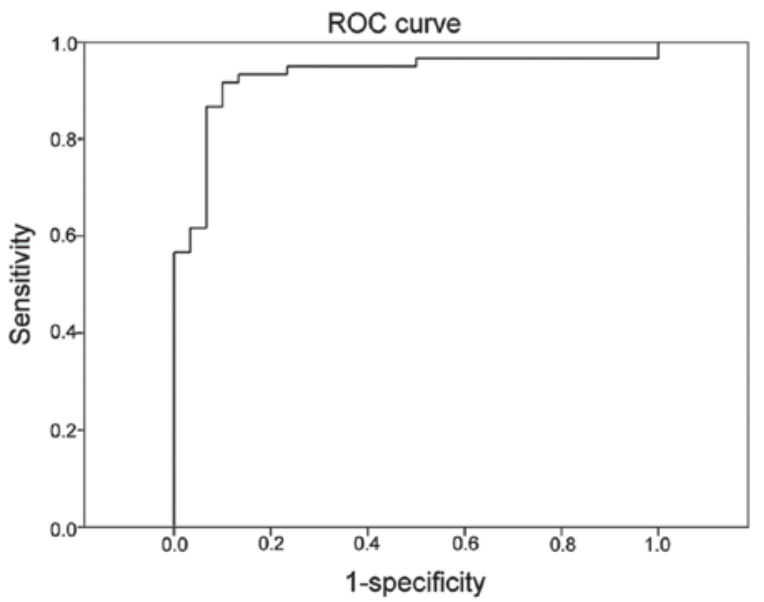

Figure 2. ROC of ELISA fluorescence by non-parameter method (fold line).

IgA, EBNA1, Zta-IgA and EA/IgA) can be used for early NPC diagnosis in the clinics (17-21). With the application of nanotechnology in biomedical filed, quantum dot emission, especially, with its unique physical and chemical advantages, small QD, high fluorescence yield, simple connection method and good stability, is used for detection on biological molecules. Moreover, QD can be used as a kind of new light-emitting marker when combined with tumor markers, which are expected to replace traditional colloidal gold for immune chromatography technology.

In this study, water-soluble carboxylated QD was combined with a crosslinking agent, EDC. The reaction principle is that - $\mathrm{COOH}$ on water-soluble carboxylated QD was activated by EDC to form a medium product, which was reacted with -NH2 on EBV EBNA1 antigen to finally form a stable covalent bond between EBV EBNA1 antigen and QD. Agarose gel 
electrophoresis showed that the stable covalent bond was formed between water-soluble carboxyl QD and EBV EBNA1 antigen by amino and carboxyl condensation on the surface. Water-soluble carboxyl QD and EBV EBNA1 antigen were successfully linked suggesting a successful labeling. QD fluorescence emission spectrum showed that emission peak of QD-EBV EBNA1 was around $380 \mathrm{~nm}$ with good fluorescence properties.

This experiment used QD immune chromatography to detect ELISA absorption value of standard NPC marker EB NA1 antibody of different concentrations. By using the ROC, the sensitivity and specificity of this technique were calculated. However, the experiment sample size is too small to determine the optimal concentration of positive standard, so whether serum sample EBV EBNA1 is negative or positive cannot be reasonably judged, and further research is needed to solve this question. Independent sample test was performed on statistical results by using Levene test. With F test $<0.1$, we can obtain the results that variance is not equal and $\mathrm{P}<0.001$, namely there is statistically significant difference between NPC group and normal control group in absorption value of serum. Therefore, it is confirmed that dual antigen sandwich method combined with QD immune chromatography is feasible, thus laying a solid foundation for further EBV EBNA1 QD immune chromatography.

Early diagnosis on malignant tumor depends on the discovery of new biomarkers, while the progress of immune detection also relies on the improvement of detection method. With the application of nanotechnology in biomedical filed, quantum dot emission, especially, with its unique physical and chemical advantages, such as small QD, high fluorescence yield, simple connection method and good stability, is used for detection on biological molecules. Moreover, it can be used as a kind of new light-emitting marker when combined with tumor markers, which are expected to replace traditional colloidal gold for immune chromatography technology. In this study, the application of QD to label NPC marker EBV EBNA1 antigen was studied among NPC patients. Combined with ELISA and immune chromatography, it is confirmed that dual antigen sandwich method combined with QD immune chromatography is feasible to detect EBV EBNA1 antibody in NPC patients. However, due to sample size and optimization of antibody standard concentration, further research is still needed to determine the detection threshold. In future studies, we are expected to develop agent kits for detection of EBV EBNA1 antibody by QD technology and develop a small-size, convenient and simple instrument. More detailed work is needed for application of QD in rapid immune detection in order to achieve QD detection with good repeatability, high credibility, and simple operation.

\section{References}

1. Yoo G, Park J, Lee SS and Sim HS: Anisotropic charge Kondo effect in a triple quantum dot. Phys Rev Lett 113: 236601, 2014.

2. Karki KJ, Widom JR, Seibt J, Moody I, Lonergan MC, Pullerits T and Marcus AH: Coherent two-dimensional photocurrent spectroscopy in a PbS quantum dot photocell. Nat Commun 5: 5869, 2014.

3. Blum AS, Moore MH and Ratna BR: Quantum dot fluorescence as a function of alkyl chain length in aqueous environments. Langmuir 24: 9194-9197, 2008.

4. Lee H, Kim C, Lee D, Park JH, Searson PC and Lee KH: Optical coding of fusion genes using multicolor quantum dots for prostate cancer diagnosis. Int J Nanomed 12: 4397-4407, 2017.
5. Nigam Joshi P, Agawane S, Athalye MC, Jadhav V, Sarkar D and Prakash R: Multifunctional inulin tethered silver-graphene quantum dots nanotheranostic module for pancreatic cancer therapy. Mater Sci Eng C 78: 1203-1211, 2017.

6. Li K, Xia C, Wang B, Chen H, Wang T, He Q, Cao H and Wang Y: Effects of quantum dots on the ROS amount of liver cancer stem cells. Colloids Surf B Biointerfaces 155: 193-199, 2017.

7. Wang Y, Li Y, Chen Z, Wang T, Gu J, Wu X, Yin Y, Wang M and Pan Z: The evaluation of colorectal cancer risk in serum by anti-DESMIN-conjugated CdTe/CdS quantum dots. Clin Lab 63: 579-586, 2017.

8. Liu Y, Zhao Y, Luo H, Liu F and Wu Y: Construction of EGFR peptide gefitinib/quantum dots long circulating polymeric liposomes for treatment and detection of nasopharyngeal carcinoma. Biochem Biophys Res Commun 490: 141-146, 2017.

9. Li LN, Xiao T, Yi HM, Zheng Z, Qu JQ, Huang W, Ye X, Yi H, Lu SS, Li XH, et al: MiR-125b increases nasopharyngeal carcinoma radioresistance by targeting A20/NF-kappaB signaling pathway. Mol Cancer Ther 16: 2094-2106, 2017.

10. Su Z, Li G, Liu C, Ren S, Deng T, Zhang S, Tian Y, Liu Y and Qiu Y: Autophagy inhibition impairs the epithelial-mesenchymal transition and enhances cisplatin sensitivity in nasopharyngeal carcinoma. Oncol Lett 13: 4147-4154, 2017.

11. Smith C, Tsang J, Beagley L, Chua D, Lee V, Li V, Moss DJ, Coman W, Chan KH, Nicholls J, et al: Effective treatment of metastatic forms of Epstein-Barr virus-associated nasopharyngeal carcinoma with a novel adenovirus-based adoptive immunotherapy. Cancer Res 72: 1116-1125, 2012.

12. Chen QY, Tang QN, Tang LQ, Chen WH, Guo SS, Liu LT, Li CF, Li Y, Liang YJ, Sun XS, et al: Pretreatment serum amyloid a and $\mathrm{c}$ - reactive protein comparing with Epstein-Barr virus DNA as prognostic indicators in patients with nasopharyngeal carcinoma: A prospective study. Cancer Res Treat: Jul 14, 2017 (Epub ahead of print). doi: 10.4143/crt.2017.180.

13. Wang C, Wang H, Zhang Y, Guo W, Long C, Wang J, Liu L and Sun X: Berberine inhibits the proliferation of human nasopharyngeal carcinoma cells via an Epstein-Barr virus nuclear antigen 1-dependent mechanism. Oncol Rep 37: 2109-2120, 2017.

14. Ramayanti O, Juwana H, Verkuijlen SA, Adham M, Pegtel MD, Greijer AE and Middeldorp JM: Epstein-Barr virus mRNA profiles and viral DNA methylation status in nasopharyngeal brushings from nasopharyngeal carcinoma patients reflect tumor origin. Int J Cancer 140: 149-162, 2017.

15. Gao R, Wang L, Liu Q, Zhang LF, Ye YF, Xie SH, Du JL, Chen SH, Guo J, Yang MJ, et al: Evaluation of seven recombinant VCA-IgA ELISA kits for the diagnosis of nasopharyngeal carcinoma in China: A case-control trial. BMJ Open 7: e013211, 2017.

16. Dobson R, Topping J and Giovannoni G: Comparison of two commercial ELISA systems for evaluating anti-EBNA1 IgG titers. J Med Virol 85: 128-131, 2013.

17. Cai YL, Li J, Lu AY, Zheng YM, Zhong WM, Wang W, Gao JQ, Zeng H, Cheng JR and Tang MZ: Diagnostic significance of combined detection of Epstein-Barr virus antibodies, VCA/IgA, EA/IgA, Rta/IgG and EBNA1/IgA for nasopharyngeal carcinoma. Asian Pac J Cancer Prev 15: 2001-2006, 2014.

18. Yang F, Liu Q and Hu CM: Epstein-Barr virus-encoded LMP1 increases miR-155 expression, which promotes radioresistance of nasopharyngeal carcinoma via suppressing UBQLN1. Eur Rev Med Pharmacol Sci 19: 4507-4515, 2015.

19. Luo YL, Chen H, Peng SG, Lin JH and Huang PY: Assessment of detection assays of Epstein-Barr viral Rta-IgG, VCA-IgA, EA-IgA and Epstein-Barr viral DNA at different clinical stages in the diagnosis of nasopharyngeal carcinoma. Zhonghua Yi Xue Za Zhi 93: 3516-3519, 2013 (In Chinese).

20. Zhang XM, Zhong JM, Tang MZ, Zhang XG, Liao J, Zheng YM, Deng $\mathrm{H}$ and Zeng Y: Comparison of IgA/VCA, IgA/EA, IgG/EA in immunoenzyme methods and ZEBRA ELISA in early diagnosis of nasopharyngeal carcinoma. Zhonghua Shi Yan He Lin Chuang Bing Du Xue Za Zhi 20: 263-265, 2006 (In Chinese).

21. Cai YL, Zheng YM, Cheng JR, Wang W, Zhang YN, Wang WH, Wu YS, Zhong WM, Li J and Mo YK: Relationship between clinical stages of nasopharyngeal carcinoma and Epstein-Barr virus antibodies Rta/IgG, EBNA1/IgA, VCA/IgA and EA/IgA. Nan Fang Yi Ke Da Xue Xue Bao 30: 509-511, 2010 (In Chinese).

This work is licensed under a Creative Commons Attribution-NonCommercial-NoDerivatives 4.0 International (CC BY-NC-ND 4.0) License. 